\title{
Posverdad y fake news en comunicación política: breve genealogía
}

\author{
Post-truth and fake news in political \\ communication: A brief genealogy
}

\author{
Raúl Rodríguez-Ferrándiz
}

Cómo citar este artículo:

Rodríguez-Ferrándiz, Raúl (2019). “Posverdad y fake news en comunicación política: breve genealogía”. El profesional de la información, v. 28, n. 3, e280314.

https://doi.org/10.3145/epi.2019.may.14

Artículo recibido el 07-09-2018 Aceptación definitiva: 18-01-2019

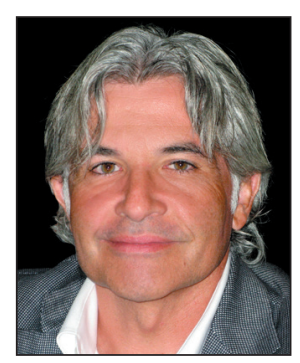

Raúl Rodríguez-Ferrándiz $\square$

https://orcid.org/0000-0003-1341-255X

Universidad de Alicante

Departamento de Comunicación y

Psicología Social

Avda. de San Vicente, s/n.

03080 Alicante, España

r.rodriguez@ua.es

\section{Resumen}

El artículo expone y discute el significado, el origen y los precedentes del término posverdad en comunicación política, así como de la expresión conexa fake news. De ambos se describen y evalúan casos recientes. Se analizan y valoran las interpretaciones que se han dado de dichos fenómenos, desde las que demuestran su continuidad (en tanto encarnaciones recientes de "demagogia», "propaganda» o "desinformación») a las que enfatizan su novedad, y desde las que ponen el acento en lo tecnológico a aquellas que hablan de una verdadera transformación epistemológica o descubren las motivaciones psicológicas que las subtienden. Finalmente, se resumen y discuten las medidas correctoras propuestas: la regulación o la autorregulación de los contenidos, el fact checking, la supervisión de los algoritmos de búsqueda, y los programas de alfabetización digital.

\section{Palabras clave}

Posverdad; Noticias falsas; Demagogia; Sociedad; Comportamiento humano; Fake news; Comunicación política; Verificación; Fact checking; Filtros burbuja; Redes sociales; Medios; Prensa; Facebook; Definiciones; Conceptos; Revisión.

\begin{abstract}
The article exposes post-truth politics and fake news meaning, origin and precedents. Recent cases of both expressions are described and discussed. We offer and value the interpretations that have been given of these phenomena, around a double axis: those that demonstrate its continuity (as novel incarnations of demagogy, propaganda or disinformation) as opposed to those that emphasize its novelty, and those that put the accent on technology versus those that speak of a true epistemological transformation or discover the psychological motivations that subtend them. Finally, corrective measures are summarized and discussed: regulation or self-regulation of contents, fact-checking, monitoring of search algorithms and digital literacy programs.
\end{abstract}

\section{Keywords}

Post-truth; Fake news; Demagogy; Society; Human behavior; Political communication; Fact checking; Filter bubble; Social networking sites; Social media; Media; Facebook; Definitions; Concepts; Review. 


\section{Introducción: la posverdad en los diccionarios}

El Oxford English Dictionary eligió post-truth como palabra del año en 2016. El término alude a aquella situación

"en la que los hechos objetivos influyen menos en la formación de la opinión pública que las apelaciones a la emoción y a las creencias personales" (OED, 2016).

El ejemplo de uso que propone es el siguiente:

"En esta era de políticas de posverdad, es fácil seleccionar datos concretos y llegar a la conclusión que desees".

En este caso el prefijo post-, como indica el diccionario, no se refiere a posterioridad en el tiempo, como en posguerra o posparto, sino a superación, cancelación o irrelevancia de aquello sobre lo que se aplica, como en post-racial o posnacional. En otras palabras, una época "posverdadera" es aquella en que la persecución de la verdad se ha vuelto inútil o quimérica. Entraríamos en una especie de suspensión voluntaria de la capacidad de juzgar los hechos por lo que son -como si averiguar cuáles son los hechos fuera tarea inútil-y una querencia por asumir "hechos" ya teñidos de color. Dado que esos colores son más vivos y parecen aportar no sólo una descripción, sino sugerir también una interpretación al hecho, permiten una justificación paradójica: es un hecho porque la interpretación lo explica de manera simple y económica (remontando el río de la lógica).

Por su parte, el Diccionario de la Real Academia de la Lengua Española registra desde diciembre de 2017, en su 23a edición, el término posverdad, que define así:

"Distorsión deliberada de una realidad, que manipula creencias y emociones con el fin de influir en la opinión pública y en actitudes sociales" (RAE, 2017).

El ejemplo sería: "Los demagogos son maestros de la posverdad". La diferencia con la definición británica merece reseñarse, más allá de que en inglés post-truth es un adjetivo y en español "posverdad" un sustantivo: donde el diccionario de Oxford define una situación (circumstance) y habla de modelaje (shaping) de la opinión pública debido a una apelación a las emociones y creencias personales, el de la Academia habla directamente de "distorsión deliberada", de "manipulación" y de "influir", de manera que la primera es más descriptiva y neutra, mientras la segunda es más valorativa y carga más las tintas. La primera no califica el modelaje ni lo atribuye a ningún interés de parte; la segunda lo descalifica como interesado y mendaz, dando por descontado que las creencias y emociones son manipulables en un sentido predeterminado y que esa es la finalidad de la tergiversación de los hechos. Por otra parte, mientras el diccionario del inglés sugiere una nota de actualidad en su ejemplo de uso (la abundancia de data y la selección de aquellos más aptos para justificar una posición), el diccionario del español emparenta la posverdad con la demagogia, de manera que tan posverdaderos podrían ser una noticia falsa producto de un burdo montaje fotográfico o una rotulación engañosa, viralizada en las redes sociales como, retrospectivamente, los sofismas dialécticos de Gorgias.

Que dos diccionarios de referencia de las dos lenguas más habladas del mundo hayan recogido el término sin duda quiere decir algo, y aquí vamos a intentar precisar qué, analizar los precedentes, la dimensión actual del fenómeno y las perspectivas que abre. Dos apuntes preliminares sobre ambas definiciones:

- Por muy venerables y solventes que sean las instituciones lexicográficas que las formulan, sería ingenuo pensar que son ajenas al contexto político, en clave nacional o internacional (el Brexit, la era Trump o el desafío independentista catalán en España) ${ }^{1}$.

- Ambas se fundan en una dicotomía o incluso una incompatibilidad entre conocimiento racional y emociones², algo que está muy lejos de ser asumido no sólo por la psicología y en general las ciencias cognitivas, sino también por la retórica, que al fin y al cabo es la primera que se ocupó de las estrategias persuasivas de la comunicación política.

Una de las paradojas de la posverdad por tanto puede formularse como sigue: ¿cómo es posible que nos sorprendamos y hasta nos escandalicemos ante un fenómeno que ha estado siempre implícito en la noción misma de "opinión pública"? Más aún, incluso la caracterización que la filosofía hizo del concepto general de "opinión" como opuesto a verdad, ¿no debería ser un precedente sólido de la posverdad? No podemos abordar aquí estas cuestiones, pero queden apuntadas. Esperamos aportar sin embargo una exposición terminológica, histórica, crítica y bibliográfica actualizada de los regímenes de la posverdad, una breve y muy modesta genealogía, en términos foucaultianos, de la posverdad.

\section{Breve historia de la posverdad}

El término "posverdad" se acuñó en los días de la Guerra del Golfo. También según el OED, fue el dramaturgo serbio-americano Steve Tesich quien empleó el término por vez primera en un artículo para The nation en 1992. En ese texto titulado "Un gobierno de mentiras", dibujaba un proceso de degradación de la calidad de la democracia y de la sociedad civil. El caso Watergate, el escándalo Irán-Contra y la Guerra del Golfo eran tres hitos en la historia reciente de EUA que demostrarían que los ciudadanos comenzaban a evitar cada vez más enfrentarse a la verdad y preferían que el gobierno les ahorrara ese trago: 
"Nosotros, como pueblo libre, hemos decidido libremente que queremos vivir en un mundo de posverdad" (Tesich, 1992).

La novedad de esta posverdad anterior a las redes que describe Tesich no sería tanto la mendacidad de los políticos como la actitud de la opinión pública ante ella, tolerante o incluso complaciente con esa ocultación de verdades incómodas.

La guerra de una coalición internacional liderada por EUA contra Irak de 2003, con la presunción de la existencia de aquellas armas de destrucción masiva jamás halladas luego sobre el terreno, pero cuyas pruebas se presentaron como consistentes, fue otro hito en la genealogía de la posverdad. Sólo un año después, en 2004, Ralph Keyes publicó un libro en cuyo título aparecía el término post-truth, aunque no cristalizó en ese momento como buzzword. Keyes sostenía que durante los mandatos de Reagan, Clinton y Bush había crecido enormemente el umbral de tolerancia de la ciudadanía y de la propia clase política con la mentira, de manera que el escándalo de otras épocas ante comportamientos deshonestos había dado paso a la indiferencia, a las excusas o los matices desculpabilizadores. Es así como habíamos llegado a la post-truthfulness:

"A pesar de que siempre ha habido mentirosos, las mentiras se solían decir con una cierta vacilación, un poco de ansiedad, una pizca de culpa, algo de vergüenza, o al menos embarazo. Ahora, haciendo uso de nuestra inteligencia, hemos encontrado razones para manipular la verdad y así poder mentir sin remordimientos. Yo lo llamo posverdad. Vivimos en una era posverdadera. La posveracidad existe en una zona de penumbra ética. Nos permite mentir sin considerarnos deshonestos" (Keyes, 2004, p. 27).

El libro de Keyes, de un tono más divulgativo que académico, coincidió con la publicación de obras más documentadas sobre el papel de la comunicación política en la guerra de Irak (Miller, 2004). Precisamente 2004 fue el año en que Tim O’Reilly popularizó el término web 2.0, y sin duda la irrupción de las redes sociales en la arena de la comunicación política, en competencia a veces, y en sinergia otras, con los medios de comunicación tradicionales, es un factor clave para explicar cómo la posverdad ha alcanzado masa crítica. Los recientes vuelcos electorales contra pronósticos y sondeos en apariencia fiables (y contra las razones políticas de un cierto establishment) se han atribuido al efecto de falsedades propaladas viralmente por dichas redes. Véase, como los casos más señalados, el referéndum sobre el Brexit (junio de 2016), el referéndum por el que fueron rechazados los acuerdos de paz con las FARC en Colombia (octubre de 2016) o las últimas elecciones presidenciales en EUA (noviembre de 2016) (Viner, 2016; White, 2017).

Los recientes vuelcos electorales contra pronósticos y sondeos en apariencia fiables (y contra las razones políticas del establishment) se han atribuido al efecto de posverdades compartidas viralmente

Con todo, hay precedentes muy autorizados de autores que sobrevolaron el concepto, sin llegar a acuñarlo, mucho antes de la era digital. Tanto George Orwell (2017 [1942]) como Alexandre Koyré (2015 [1943]) se refirieron a la mentira en el ámbito del discurso político, con el trasfondo de la recientemente acabada Guerra Civil española en el primer caso, y de la II Guerra Mundial en pleno apogeo en el segundo. El primero de ellos pasa por ser quien pergeñó de manera más sólida el concepto de fake news y lo ilustró en la historia real de su tiempo ${ }^{3}$, años antes de emplearlo como ingrediente de su más conocida ficción distópica. Ya en los años sesenta y setenta Hannah Arendt nos dejó unas agudas reflexiones sobre la verdad y la mentira en la política, cuya lectura actual es sin duda tan iluminadora como estimulante, y que tienen como marco la posguerra mundial y la Guerra del Vietnam (Rodríguez-Ferrándiz, 2018a). En ellas caracteriza la "mentira moderna" (la desaparición de Trotski de la historia oficial soviética, por ejemplo) con respecto a la "mentira tradicional", y afirma:

"El resultado de una constante y total sustitución de la verdad de hecho por las mentiras no es que las mentiras sean aceptadas en adelante como verdad, ni que la verdad se difame como una mentira, sino más bien que el sentido por el que nos orientamos en el mundo real -y la categoría de la verdad versus la falsedad está entre los medios mentales para alcanzar este fin- queda destruido. Y para este problema no hay remedio" (Arendt, 2017, pp. 68-69 [1964 y 1971]).

Por su parte, Norman Mailer en su biografía de Marilyn Monroe acuñó en 1973 el término factoid, que definió como

"hechos que no existen antes de aparecer en una revista o periódico, creaciones que no son tanto mentiras como un producto para manipular emociones" (Mailer, 1973).

Más de una década después, en 1985, e intrigado por el término, el profesor de lingüística David Irving Shapiro requirió a Mailer para que lo precisara, a lo que el periodista y escritor respondió lo siguiente:

"La palabra factoide se usaba para dramatizar el hecho, y es un hecho, no un factoide, que un vasto porcentaje de lo que tomamos por real, codificado, observado, verificado y de facto verdadero, de hecho, no se basa más que en su existencia en letra impresa. No hace falta decir que, una vez que se imprime un factoide, se reimprime en muchos otros periódicos, servicios de cable, etc., y adquiere una realidad psicológica a menudo más poderosa que la otra realidad psicológica asociada al hecho real" (Lennon, 2014, pp. 594-595).

De manera que la viralidad del factoide en los teletipos y en la prensa escrita prefigura sin duda la de la posverdad en las redes sociales, ambos con una vida en el papel o en las pantallas, sin correlato en la realidad, pero con la inquietante 
capacidad de hacerse realidad: la mera enunciación del fake como profecía autocumplida, en la línea de esos pseudo-events de los que había hablado unos años antes Daniel Boorstin (1991 [1961]). Mailer fue uno de los representantes y teórico además del "nuevo periodismo", de manera que su crítica de los factoides parece una especie de cordón sanitario para proteger las prácticas de ese estilo de escritura de no-ficción (o de faction, como fue llamada en una feliz palabra-maleta) de esos otros rumores sin consistencia, de esas dramatizaciones o espectacularizaciones de un suceso acaso banal, destinado a ponerlo en órbita en los medios.

Más recientemente, Harry Frankfurt (2006) reflexionó sobre la palabra bullshit, que tiene matices de sentido que la apartan de lie, y que la vuelven difícilmente traducible: mientras el mentiroso reconoce y hasta honra perversamente la verdad, tergiversándola pero teniéndola en mente y en cierto modo también en aprecio, el bullshitter vuelve irrelevante la diferencia entre verdad y mentira: o bien no domina el tema sobre el que habla, y entonces su discurso es una cháchara insustancial, o bien es escéptico sobre la posibilidad de hallar la verdad, y sustituye la corrección de sus juicios por la sinceridad, es decir, una verdad circunscrita y subjetiva.

Justo antes del bautismo del término post-truth por el OED, y sin llegar a utilizarlo, Mark Thompson (2016) recuperó el concepto de "autenticismo" y lo identificó como ingrediente del discurso político que triunfa hoy: simplicidad expresiva que sugiere honradez emocional, sospecha de los meros hechos, que son fríos, anecdóticos, estadísticos y emborronan o distraen de las "verdades" que laten debajo, enfático desprecio de la retórica, todo lo cual no impide explicar en términos precisamente retóricos la operación. Thompson disecciona con precisión estilos de comunicación política y discursos o declaraciones públicas de Lyndon Johnson, Nixon, Thatcher, Blair, Cameron, Reagan, los dos Bush, los dos Clinton, Sarah Palin y Barack Obama, además del propio Trump cuando era todavía candidato. También analiza la potencia mendaz de lemas como "cambio climático" (en vez de "calentamiento global”), "armas de destrucción masiva", "comité de la muerte" (contra la subvención de la eutanasia que contemplaba el Obamacare), etc.

\section{Fake news como instrumento de la política de la posverdad}

Una expresión casi indisolublemente ligada a la de posverdad es la de fake news, elegida como palabra del año en 2017 por el Collins Dictionary, que la define como

"información falsa, a menudo sensacional, difundida bajo el disfraz de noticia”.

Por su parte, el Cambridge Dictionary precisa en su entrada

"historias falsas que parecen ser noticias, difundidas en internet o usando otros medios, generalmente creadas para influir en puntos de vista políticos o como una broma".

El ejemplo que pone este último diccionario es:

"Existe preocupación sobre el poder de las noticias falsas de afectar los resultados electorales"

El informe anual que publica Reuters sobre la información en la Red recoge en su edición de 2017 que la expresión puede referirse a:

- "Noticia que se 'inventa' para ganar dinero o desacreditar a otros;

- noticia que se basa en hechos, pero se crea para adaptarse a una agenda particular; y

- noticia con la que la gente no se siente cómoda o no está de acuerdo".

Por su parte, el Oxford Institute for the Study of Computational Propaganda define las fake news como

"información engañosa o incorrecta, que pretende ser una noticia real sobre política, economía o cultura" (Har$\sin , 2018)$.

Como ya vimos a propósito de "posverdad", las definiciones y los ejemplos oscilan entre una caracterización neutra, digamos atemporal (Collins Dictionary, Oxford Institute for the Study of Computational Propaganda), y otra vinculada específicamente a la gestión de la información y a la economía de la atención en la era de las redes sociales (Cambridge English Dictionary; Bounegru et al., 2017).

Una nota distintiva con respecto a posverdad es que algunos diccionarios y autores vinculan el empleo del término fake news con la manipulación de una noticia auténtica con un afán humorístico, en un marco que pretende asegurarse de que es percibido el tono satírico o paródico. De hecho el término se registra como marca explícita por vez primera en el programa de la televisión norteamericana The daily show, presentado por Jon Stewart (1999-2015), de la cadena de pago Comedy Central. Es decir, un programa abiertamente rotulado y consagrado a la sátira política en una cadena temática de humor. En España, secciones humorísticas de la prensa como Tremending topic de El diario, o periódicos online como El mundo today serían ejemplos equivalentes. Ahora bien, ese sentido debe ser distinguido, como parece evidente, de aquellas piezas pretendidamente informativas que también imitan formalmente la apariencia de géneros bien conocidos (noticia, reportaje,

Las definiciones de posverdad oscilan entre una caracterización neutra o atemporal, y otra vinculada específicamente a la gestión de la información y la atención en la era de las redes sociales 
entrevista), pero que tienen el propósito malicioso de desinformar sobre asuntos políticos, porque aspiran a ser tomadas por fidedignas o al menos plausibles (McNair, 2018). Y que pueden reportar por otra parte cuantiosos ingresos (Harsin, 2018).

Más allá de la etiqueta como meme de plena actualidad, McNair señala que

"las noticias siempre han sido criticadas por ser parciales, plagiadas, tergiversadas, fabricadas, ficticias y, sí, falsificadas",

recordando el titular de una revista en 1925 que rezaba "Fake news and the public", y advertía de los efectos perniciosos de una nueva tecnología como el telégrafo en la diseminación de patrañas (McNair, 2018, p. 17). Este autor cita decenas de casos habidos desde entonces, en los que las noticias falsas fueron destapadas, los reporteros responsables apartados y las rectificaciones debidamente publicadas. Para McNair la situación a día de hoy difiere en que no hay voluntad de restituir la objetividad cuando ha sido vulnerada, porque la carga de la prueba se vuelve precisamente contra ese paradigma de objetividad, atribuida a una fabricación del establishment, y porque la velocidad del entorno informativo digital lleva a un infochaos en el que el arma arrojadiza de la controversia política es precisamente acusar al rival (sea un medio de comunicación o un actor político) de propalar fake news. Pero las fake news, para este autor, convendría distinguirlas cuidadosamente no sólo de las parodias explícitas, sino también del sesgo político partidista y su bien conocido recurso a lo capcioso. Las fake news son

"desinformación intencionada (invención o falsificación de hechos conocidos) con fines políticos y/o comerciales, presentada como noticias reales" (McNair, 2018, p. 38).

Y tiene unas causas complejas, a la vez filosóficas y epistemológicas (relativismo rampante), culturales (declive de la confianza en las élites), económicas (monetización de la información en situación de aguda competencia por los ingresos publicitarios asociados), tecnológicas (proliferación de plataformas que difunden información) y políticas (auge del nacionalismo y el populismo, cuyo epítome sería Trump).

En esta línea, la irrupción de las fake news en la controversia pública reciente se produjo cuando afloraron sospechas fundadas de graves injerencias de una "fábrica" de estos productos tóxicos radicada en San Petersburgo, la Agencia Rusa de Investigación de Internet, en la campaña presidencial norteamericana de 2016. Dichas piezas desinformativas habrían influido en amplias franjas del electorado estadounidense a través de Facebook principalmente, y habrían distorsionado el debate público a favor de Trump, denigrando a Hillary Clinton y pro-

Las fake news se atribuyen a fenómenos filosóficos (relativismo rampante), culturales (declive de la confianza en las élites), económicos (monetización de la información), tecnológicos (proliferación de plataformas) y políticos (auge de nacionalismo y populismo)

pagando la discordia general, aspectos que recogió ya el 6 de enero de 2017 la US Intelligence Community, una federación de dieciséis agencias gubernamentales de inteligencia en relaciones internacionales y en seguridad nacional. En mayo de 2018 el Senado norteamericano validó las conclusiones de dicha macroagencia (Demirjian, 2018; Petit, 2018).

De hecho según buzzfeed.org, en los tres meses de campaña previos a las elecciones en EUA, los sitios fraudulentos y los blogs extremadamente radicalizados y partidistas (como Breitbart, Right Wing News y Freedom Daily) que difundieron noticias falsas de interés electoral superaron en impacto en Facebook a las noticias electorales auténticas generadas por medios acreditados como The New York Times, The Washington Post, NBC News y otros. Entre las primeras, que el Papa Francisco I apoyaba abiertamente la candidatura de Trump, que WikiLeaks desveló que Hillary Clinton había vendido armas al ISIS, que el líder del ISIS pidió el apoyo de los musulmanes americanos a Clinton y que un operario de mantenimiento descubrió en un almacén en Columbus (Ohio) decenas de urnas repletas de votos para Clinton. Las veinte primeras noticias falsas sumaban 8,7 millones de impactos en Facebook (reacciones, compartir, comentarios), mientras las veinte primeras de diarios de referencia sólo alcanzaban 7,3 millones (Silverman, 2016; Silverman; Singer-Vine, 2016) ${ }^{4}$

Twitter tampoco es ajeno a esta ceremonia de la confusión. Los investigadores del Project on Computational Propaganda de la University of Oxford mencionado arriba midieron la actividad de bots y "noticias chatarra" (junk news ${ }^{5}$ es el término que utilizan, y que prefieren al de fake news) en Twitter entre los votantes de Michigan -un estado electoral clave- en los días previos a las elecciones presidenciales de EUA en noviembre de 2016, realizado sobre 140.000 tweets. Determinaron que alcanzaba casi la cuarta parte del total de contenidos (el 23\%) e igualaba al de noticias "profesionales" de medios acreditados (Howard et al., 2017; Howard; Wooley; Calo, 2018). El mismo equipo de investigación había analizado un año antes los mensajes en Twitter en torno a hashtags pro y contra la salida de Gran Bretaña de la Unión Europea. Sobre un corpus de más de millón y medio de tweets publicados entre el 5 y el 12 de junio de 2016, que circularon entre más de 300.000 cuentas de Twitter, comprobaron que sólo un 1 por ciento de las cuentas eran responsables de la tercera parte del tráfico, y que dichas cuentas publicaban o retweeteaban hasta 50 tweets al día, es decir, a un ritmo difícilmente al alcance de un operador humano. Se trataba en conclusión de bots o mensajes automatizados que imitan una interacción humana, y que en conjunto se mostraban mucho más proclives a la posición \#Brexit o \#voteleave que a la posición \#Strongerln o \#voteremain, en una proporción de 3 a 1 (Howard; Kollanyi, 2016). 
Como ejemplo todavía más reciente merece la pena recordar el caso Cambridge Analytica. The New York Times y The observer destaparon conjuntamente en marzo de 2018 este escándalo de acceso indebido a decenas de millones de perfiles de Facebook, a uno y otro lado del Atlántico. Todo partió de una inocente encuesta con una finalidad supuestamente académica, respondida por sólo unos cientos de miles de usuarios en Facebook (que sí accedieron a compartir sus datos), y que llevó a que los de ochenta y siete millones de ellos llegaran a una empresa británica, Cambridge Analytica. Dichos datos se utilizaron para microtargeting digital enfocado a citas electorales, en particular a afinar y personalizar la comunicación referida al referéndum del Brexit y a las presidenciales norteamericanas, lo cual incluía -y este es el aspecto más inquietante del asunto- el empleo de piezas informativas manifiestamente fraudulentas, o anuncios que resonaban con las concretas inclinaciones de los receptores. Al parecer Cambridge Analytica dispuso de perfiles psicográficos de los usuarios, basados en el modelo Ocean, que representa cinco rasgos de personalidad:

- Apertura: openness, receptividad al cambio y a las experiencias novedosas;

- Conciencia: tendencia a la perfección;

- Extraversión: sociabilidad y deseo de estar próximo a los demás;

- Amabilidad: empático y cooperativo;

- Neuroticismo: susceptibilidad, facilidad para sentirse molesto o ansioso.

El experto en psicometría de la Stanford University Michał Kosiński elaboró en 2008 un protocolo para analizar a los usuarios de la Red a partir de su actividad en Facebook. Solo tenía la intención de enviarlo a unas pocas decenas de sujetos para obtener resultados de investigación, pero luego se vio abrumado por el índice de respuestas de su encuesta, que se viralizó y fue respondida por 58.000 informantes. En 2013 logró demostrar que, sobre la base de un promedio de 68 likes otorgados en Facebook por un usuario, era posible predecir su color de piel (95\% de precisión), su orientación sexual (88\%) y su tendencia política (demócrata o republicano, con un 85\%) (Kosiński; Stillwell; Graepel, 2013). Kosiński rechazó la oferta de integrarse en una empresa, Strategic Communication Laboratories Group (SCL Group), radicada en Londres, dedicada a estrategias de difusión de información política, campañas sociales, así como puramente comerciales. Cambridge Analytica fue precisamente una spin-off the SCL Group. Entre sus directivos, Robert Mercer, multimillonario y donante generoso en las campañas republicanas, que fue el fundador de la empresa, y Steve Bannon, hasta hacía poco estratega jefe de Trump, que era su vicepresidente. En 2016, la empresa pudo haberse hecho con hasta 220 millones de perfiles de personalidad basados en pruebas Ocean, así como paquetes brutos de datos personales comprados a terceros, como Acxiom y Experian (Cadwalladr; Graham-Harrison, 2018; Rosenberg; Confessore; Cadwalladr, 2018).

La relevancia del fenómeno y la alarma social resultante se evidencia en numerosos documentos e iniciativas de instituciones de todo el mundo, como:

- el informe Can public diplomacy survive the internet? Bots, echo chambers, and disinformation, de la Advisory Commission on Public Diplomacy (Departamento de Estado de los EUA). En él se enmarca la cuestión de las fake news y el uso de algoritmos y otras herramientas de la inteligencia artificial, y varios expertos dictaminan sobre la problemática de los bots, las burbujas informativas o las cámaras de resonancia (filter bubbles o echo chambers, Del-Vicario et al., 2016; Pariser, 2011; Andrejevic, 2013) y la desinformación en los procesos políticos (Powers; Kounalakis, 2017);

- el Consejo de Europa encargó un informe, Information disorder: Toward an interdisciplinary framework for research and policy making, publicado en septiembre de 2017 (Wardle; Derakhshan, 2017);

- la Comisión Europea publicó en 2018 A multi-dimensional approach to disinformation. Report of the Independent High Level Group on Fake News and Online Disinformation (European Commission, 2018);

- la Unesco se ha sumado al coro de quienes advierten del peligro y ofrecen recetas: Journalism, 'fake news' \& disinformation handbook for journalism education and training (Ireton; Posetti, 2018).

En todos ellos se evidenciaba el nuevo desorden informativo, se alertaba contra el efecto de los algoritmos que personalizan las búsquedas y los resultados obtenidos y determinan la experiencia de navegación de cada usuario, y se identificaban los fact checkers más solventes, las estrategias de los poderes públicos y de los operadores de redes o de medios privados y las tácticas de lectura de los propios usuarios que eventualmente podrían revertir la situación.

Las propias redes sociales han asumido el problema y se han apresurado a proponer medidas. Google informó de que las páginas con noticias falsas ya no podrían usar su servicio de publicidad, para así dejarlas sin ingresos, y anunció que modificaría sus algoritmos para evitar que escalaran posiciones en las búsquedas de los usuarios (Wingfield; Isaac; Benner, 2016). Facebook también adoptó una medida similar, y en abril de 2016 publicó en los newsfeeds de los usuarios una guía para identificar noticias falsas. Algunos de los consejos eran de sentido común, y otros fácilmente falseables por quien tuviera el propósito de mentir y dedicara los suficientes recursos para hacerlo: ser escéptico con los titulares muy llamativos, mirar bien la URL de las webs, investigar la fuente, fijarse en si se usa un formato inusual, comprobar las evidencias mencionadas, comparar el artículo y su información con otros artículos y, finalmente, ser crítico y sólo compartir las noticias fiables (Sulleyman, 2017).

Con todo, algunos trabajos de campo advierten de que la alarma social y la de la clase política resulta injustificada. El Reuters Institute for the Study of Journalism (RISJ, de la University of Oxford, principal centro de excelencia que ha analizado el fenómeno de las fake news, afirma en su informe Measuring the reach of "fake news" and online disinformation in Europe (febrero de 2018) que las fake news tienen un alcance más limitado de lo que algunas veces se piensa. El 
informe, que analiza esta mala praxis en Francia e Italia a lo largo de 2017, afirma que ninguna de las webs analizadas que difundían noticias falsas tenía en 2017 una audiencia mensual superior al 3,5\%. Las que tenían una mayor audiencia no llegaban al $1 \%$ de la población online, tanto de Francia como de Italia. En comparación, las webs de información más populares en Francia (Lefigaro.fr) e Italia (Larepubblica.it) tenían una audiencia mensual media del 22,3\% y del 50,9\%, respectivamente. Además, en la mayoría de los casos, tanto en Francia como en Italia, la difusión de fake news en redes sociales no genera tantas interacciones como los medios y marcas informativas establecidas (Fletcher et al., 2018). Otro estudio del RISJ también relativiza la importancia de las redes sociales. Una investigación realizada en nueve países (Alemania, Australia, Dinamarca, España, Estados Unidos, Francia, Grecia, Irlanda y Reino Unido) concluye que en materia informativa, las redes sociales obtienen la confianza del $24 \%$ de los usuarios frente al $40 \%$ de los medios tradicionales (Petit, 2018).

Eso está en línea con análisis independientes basados en evidencias empíricas obtenidas en Estados Unidos (Allcott; Gentzkow, 2017; Guess; Nyhan; Reifler, 2018; Nelson; Taneja, 2018). Según diversos trabajos sobre la difusión de noticias falsas en las elecciones estadounidenses de 2016, las redes sociales sólo fueron la principal fuente de información para el $14 \%$ de la población, de manera que el riesgo de burbujas informativas, fake news y sesgos en los algoritmos se ve compensado por la cultura en medios de cada país. Durante el período de octubre-noviembre de 2016, los meses inmediatamente anteriores a las elecciones en EUA, el consumo de noticias falsas se concentró en un grupo pequeño. Casi 6 sobre 10 visitas a webs con noticias falsas analizadas provenía del 10\% de personas con dietas de información online ultraconservadoras. El consumo se reduciría pues a un grupo pequeño de usuarios de internet muy activos (Petit, 2018). Frente a esta posición, y sin entrar a valorar el cambio en la tendencia de voto de los usuarios alcanzados por las noticias falsas, hay estudios que concluyen que no es una adscripción partidista radical la que lleva a compartir mensajes tendenciosos o fraudulentos, sin comprobación previa de su veracidad, sino la pereza (Pennycock; Rand, 2018). Esa línea es consistente con los estudios que afirman que la mayor parte de los usuarios no se preocupan ni comprueban la fuente de la noticia que han recibido a través de las redes sociales (y que luego comparten a su vez) (Moses, 2016).

Por otro lado, los datos del Pew Research Center, según algunos, han sido malinterpretados en medios periodísticos y por algunos investigadores. Will Oremus precisa que el hecho de que la mayoría de los estadounidenses (62\% en 2016) obtengan de las redes sociales información política debe ser ponderado por el dato de que sólo el $18 \%$ realmente se nutre activamente de las noticias en redes sociales, mientras ese $62 \%$ incluye a quienes ven noticias en su newsfeed sin haberlas buscado ex profeso (Oremus, 2016). Las investigaciones del Reuters Institute for the Study of Journalism en varios países han dado resultados similares a los del Pew Research Center. El 51\% de los participantes del estudio (una encuesta a más de 50.000 personas en 26 países en el año 2016) afirma que usa las redes sociales como fuente de noticias al menos una vez por semana, pero sólo el $12 \%$ afirma que son su fuente principal.

Ciertamente, frente a las fake news se han alzado los fact checking sites, que se esfuerzan en contrastar la información y evidenciar las falsedades. Snopes, PolitiFact (EUA), Full Fact (GB) y Maldito Bulo (España) son sitios web promovidos desde instancias ajenas a los medios tradicionales, pero dedicados a un periodismo de datos

\section{Frente a las fake news se han alzado los fact checking sites, que se esfuerzan en contrastar la información y evidenciar las falsedades}

que destapa rumores insidiosos que circulan por la Web. Hay periódicos que han implantado sus propios mecanismos de comprobación, algunos con notable éxito, como el FactChecker de The Washington Post, que contabiliza de manera rigurosa las falsedades presentes en el discurso del presidente Trump (Fact Checker, 2017; 2019), pero también Désintox (Libération); Décodeurs (Le monde); La chistera (El confidencial); El cazabulos (El diario) o Hechos (El país).

Incluso cadenas de televisión, como Channel 4 (Fact check) o la BBC (Reality check) en GB, la ARD en Alemania (Faktenfinder) o La sexta en España (El objetivo) (Ufarte-Ruiz; Peralta-García; Murcia-Verdú, 2018). La eficacia de los comprobadores de hechos en la corrección de las misperceptions está en discusión: mientras algunos sostienen que en ciertos casos refuerzan más que corrigen aquello cuya veracidad discuten, es decir, exacerban el sesgo de confirmación de quien había asumido el fake (el backfire effect, Nyhan; Reifler, 2010; Guess; Nyhan; Reifler, 2018), otros destacan su capacidad para revertir la situación y servir a los fines de una pedagogía informativa (Porter, 2017).

\section{Interpretaciones de la posverdad}

Las interpretaciones sobre la posverdad basculan en general entre dos posiciones. Hay quienes la juzgan un término de moda que ha acertado a etiquetar un procedimiento muy antiguo, conocido desde la retórica clásica (demagogia podría ser uno de sus precedentes, como sugiere el $D R A E$ ) y, por no remontarnos tanto, bien identificado ya en la prensa de finales del siglo XIX y principios del XX (propaganda, podría ser el término en este caso, o desinformación) (Pickard, 2016; Floridi, 2016; Cooke, 2017; Stanley, 2017, European Commission, 2018).

En cambio, hay quienes juzgan que su irrupción responde a factores inéditos. Unos los atribuyen a los efectos casi inevitables de la telemática, la red 2.0 y del predominio de los datos sobre los hechos (Davies, 2016; Del-Vicario et al., 2016; D’Ancona, 2017). Según éstos, sería el precio que debemos pagar por la fragmentación de los proveedores de información, antes fuertemente centralizados, por el empoderamiento comunicativo del usuario y por la sobreabun- 
dancia y aceleración informativas resultante, que la volvería inmanejable y socavaría la posibilidad del acuerdo hasta en la exposición de los hechos.

Algunos sugieren que las redes sociales promueven una confusión rentable entre el dominio privado y el público, de manera que el tono de confidencia y la revelación de lo íntimo tiñen la información política y económica, intercambiando veracidad por sinceridad. La pasmosa inversión afectiva del usuario en friendship y reliability, que le lleva a la confesión de tendencias, aspiraciones, deseos, aficiones, afiliaciones y debilidades, le vuelve más receptivo en esa precisa interfaz telemática de su perfil en la red social de su elección, ante noticias de índole comercial o política, que sin embargo habría rechazado en otro medio como anuncios publicitarios o burda propaganda electoral (Kennedy, 2013; Van-Dijck, 2013; Fuchs, 2014).

Otros autores no lo achacan tanto a determinismos tecnológicos, como a razones epistemológicas (un cambio en el régimen de la verdad, Harsin, 2015), o bien a motivaciones psicológicas profundas en la construcción de la ideología mediadas, pero no determinadas, por la tecnología (Chis, 2016). En cuanto a la primera explicación, Harsin señala que el nuevo panorama revela la existencia de "mercados de la verdad": la verdad es una empresa cotizada, una operación o función que depende de variables, su circulación se gestiona en algoritmos que definen nichos de mercado. No es una verdad única, se trata de verdades plurales, estadísticas, diseñadas a medida del consumidor, un consumidor que ha suministrado, a través de su interacción social en redes, tal cantidad de datos (con inclinaciones, preferencias, gustos, quejas y rencores) que la verdad que le aparece en pantalla está personalizada y, por tanto, se hace fácil de comprar en cada caso. Es una verdad asumible, "adquirible", resulta creíble y fiable porque viene a adecuarse al retrato robot psicológico que nosotros mismos proporcionamos. Nuestras interacciones en la Red no sólo dibujan nuestro perfil consumidor, sino ciudadano, político, de manera que esos algoritmos refinados, que nosotros alimentamos con datos incesantes, diseñan patrones de conducta, previsiones sobre nuestra predisposición a asumir ciertas verdades.

Mientras los regímenes de la verdad del siglo XX corresponden a la sociedad disciplinaria o de la vigilancia que definió Foucault, donde figuraban "árbitros de la verdad" bien reconocibles (1976, p. 112), estos nuevos regímenes de la posverdad corresponden a una sociedad del control (Deleuze, 1990), en la que el poder explota las nuevas libertades que ha concedido a los individuos: la de participar-producir-expresarse y la de consumir-difundir-evaluar.

En ese escenario asistimos a una proliferación de falsedades y mentiras (fakes, hoaxes), rumores maliciosos, tergiversaciones de los hechos, que llevan aparejado un esfuerzo equivalente de fact checking, de rumor-debunking, todo ello dirimido en gran parte en las redes sociales, con la particularidad de que esos esfuerzos por rescatar la verdad o denunciar las mentiras no encuentran una autoridad lo suficientemente poderosa para imponerse, no hay ninguna institución legitimada para suscribir un contrato fiduciario sobre la verdad de los hechos. La polémica científica, social, política y económica sobre el calentamiento global es una buena muestra.

En cuanto a la segunda explicación, Chis (2016) apunta a la inescapable condición de constructos sociales de los algoritmos y de los filtros burbuja derivados, que no son productos de una caja negra tecnológica y en cierto modo inhumana, que actúa de manera ciega y compulsiva, y de la que podríamos escapar cancelando nuestros perfiles en las redes sociales. Los algoritmos materializan o son los instrumentos de pulsiones psicológicas profundamente humanas: afinidades electivas que traducen motivos relacionales, epistémicos y existenciales que responden a su vez a necesidades de esos tres órdenes (y que, dicho sea de paso, "padecen" los propios programadores humanos de estos algoritmos). Así, es natural que los individuos deseen:

- afiliarse con otros individuos en relaciones interpersonales capaces de proporcionarles identidad social, solidaridad y una porción de realidad compartida (motivos relacionales);

- reducir la incertidumbre, la complejidad o la ambigüedad y buscar apoyo cognitivo en certezas, estructura y orden (motivos epistémicos);

- gestionar las circunstancias adversas amparándose en la seguridad, en la autoestima y en la búsqueda de un sentido a la propia vida (motivos existenciales).

La arquitectura algorítmica de las redes sociales hace de caja de resonancia de estas motivaciones psicológicas. Los filtros burbuja sólo exacerbarían estas tendencias bien conocidas, aunque su generalización y refinamiento pueden ser altamente perjudiciales para la vida en democracia, porque polarizan y evidencian en cierto modo la peor faz del término "ideología", al decir de Mannheim:

"Cuando el término denota que somos escépticos con respecto a las ideas y representaciones avanzadas por nuestro oponente. Se consideran disfraces más o menos conscientes de la naturaleza real de una situación, cuyo verdadero reconocimiento no estaría de acuerdo con sus intereses. Estas distorsiones van desde mentiras conscientes hasta disfraces medio conscientes e inconscientes; desde intentos calculados para engañar al otro hasta el autoengaño" (Mannheim; Wirth; Shils, 1936). 


\section{Discusión y conclusiones}

La posverdad como estrategia de comunicación política plantea graves problemas: no es el menor de ellos la responsabilidad de los nuevos mediadores digitales en la conformación de un campo de juego limpio para la información política fiable (Enguix-Oliver, 2017). Si la irrupción de cantidades ingentes de fake news la reconocen no sólo los analistas, los investigadores y los periodistas, sino también las propias redes sociales que son el soporte a través del cual se viralizaron y se monetizaron (Jiménez-Cano, 2017a; 2017b; 2017c; Mars; Jiménez-Cano, 2017; Faus, 2018), entonces alguna medida correctora hay que tomar. Se han propuesto varias (Marda; Milan, 2018): regulación gubernamental, autorregulación, supervisión de terceros independientes, control de los parámetros de los algoritmos de búsqueda y de jerarquización de contenidos, fact checking y alfabetización en nuevos medios o específicamente en information literacy (Eisenberg, 2008; Elmborg, 2006).

La cuestión parece radicar en el factor tecnológico, que para algunos sería clave a la hora de provocar un salto cualitativo entre las armas de persuasión de la propaganda y las estrategias de desinformación bien conocidas y la situación actual, que ha sido llamada de computational propaganda. Ésta puede ser definida como una especie de tormenta perfecta consistente en la coincidencia de plataformas de redes sociales, agentes autónomos y big data, dirigidos a la manipulación de la opinión pública (Woolley, 2017). Los bots de redes sociales son las principales herramientas utilizadas en la difusión de la propaganda computacional y sirven para automatizar el compromiso político con el fin de manipular la opinión pública. Permiten la amplificación masiva de puntos de vista políticos, empoderando a un pequeño grupo de personas para establecer la agenda de conversación online. Cuando están en manos de actores

Regulación gubernamental, autorregulación, supervisión de terceros independientes, control de los algoritmos de búsqueda y jerarquización de contenidos, fact checking e information literacy son algunas medidas propuestas para contrarrestar la posverdad

políticos poderosos y dotados de recursos, estas herramientas automatizadas pueden utilizarse tanto para impulsar como para silenciar la comunicación y la organización entre los ciudadanos, tanto en los regímenes democráticos como en los autoritarios. Los expertos han calculado que más del 10\% del contenido en los sitios web de las redes sociales y el $62 \%$ del tráfico web es generado por bots. Los robots dominan muchas tareas cotidianas en internet, desde servir a la generación de preferencias personales de noticias online, hasta el display de publicidad, promover perfiles en las plataformas de redes sociales e imitar por tanto a usuarios reales. En los últimos cuatro años, numerosos medios de comunicación, desde The New York Times hasta The guardian, han tratado en sus cabeceras el creciente uso de bots. Intentan explicar cómo funcionan estos scripts automatizados con orientación social en contextos específicos, desde las citas privadas online hasta el intercambio de anuncios en tiempo real. Sin embargo, las formas en que se despliegan los bots están evolucionando más allá de las esferas sociales (una "socialidad" entendida en el sentido de compartir lo privado, lo íntimo, al modo en que lo entiende por ejemplo Facebook, Van-Dijck, 2013; Fuchs, 2014) hacia aquellas específicamente políticas. Ciertas instancias políticas, gobiernos y corporaciones habrían comenzado a utilizar esta variedad de software bot para manipular la comunicación y el compromiso, enfriar el debate o polarizarlo a conveniencia y enturbiar los problemas políticos (Wooley, 2017).

Pero de la misma manera que, como vimos, no podemos achacar la inusitada viralidad y virulencia de las fake news en la era de la posverdad a factores sólo tecnológicos, ni tampoco específicamente políticos (McNair, 2018), tampoco las soluciones pueden incidir sólo en estos aspectos. Si hay un cambio epistemológico que afecta al régimen de la verdad imperante, y si hay motivaciones psicológicas bien documentadas, de carácter emocional, epistémico y existencial, que explican las afinidades electivas que guían nuestras interacciones a través de la Red, entonces la cuestión no se resuelve ejerciendo un control experto y honesto sobre los algoritmos y promoviendo guías sobre el uso y abuso de la información en internet.

Por otro lado, la determinación del valor de verdad de todos los contenidos que circulan por las redes y que se hacen pasar por noticias exigiría consensuar una autoridad validadora, dedicar ingentes recursos a la determinación de la fiabilidad y arriesgarse a denuncias de censura. Lo más paradójico de la situación, y que debería mover a reflexión, es que esta alarma social en torno a las fake news sucede en una época que produce y difunde ingentes cantidades de "datos" y los enarbola como si fueran emanaciones de la verdad. Una época que ha consagrado la "transparencia" como gran imperativo no sólo de todo lo público, sino de la publicidad de todo lo privado. Una época, en fin, que ha hecho de la cultura del compartir un imperativo categórico (disclosure, sharing and friending como totems, Rodríguez-Ferrándiz, 2018b). Todo lo cual sugiere que una mayor exigencia de transparencia, una mayor abundancia de datos y una mayor presión por compartirlos (ya seleccionados e interpretados por instancias no sometidas a escrutinio) no esclarece más el asunto de que se trata, ni proporciona el ciudadano más elementos de juicio racional. La cuestión es precisar qué parte de la política de la posverdad es una estrategia humanísima, y por lo tanto intemporal y en cierto modo fatal, y qué parte está auxiliada por una tecnología del procesamiento y la gestión de datos sobrehumanamente refinada, y cómo podemos intervenir sobre esta última de una manera no sólo tecnológica, sino cívica, jurídica, pedagógica y económica.

La cuestión es precisar qué parte de la política de la posverdad es una estrategia humanísima, intemporal y en cierto modo fatal, y qué parte está auxiliada por una tecnología del procesamiento y la gestión de datos sobrehumanamente refinada 


\section{Notas}

1. De hecho, en la misma actualización del diccionario de Oxford donde apareció "post-truth" también lo hizo el término "Brexiteer". Por otro lado, el 18 de diciembre de 2017, sólo unos días antes de que el DRAE lanzara la nueva edición y los términos nuevos más significativos, la entonces vicepresidenta del Gobierno de España Soraya Sáenz de Santamaría declaró en el Senado que el proceso catalán había sido "un fake en toda regla, un proceso basado en la posverdad, donde las falsedades no sólo viajaban por la Red, sino también en coche oficial" (Coromina; Padilla, 2018). Finalmente, postruth y el otro término conexo, fake news, se han convertido también en armas arrojadizas en la controversia política que enfrenta, desde la campaña electoral, a la administración Trump y a varios medios informativos de EUA (en particular, The New York Times, The Washington Post y la CNN), de manera que unos acusan a otros de fabricar posverdades en forma de fake news (Bump, 2017; Cillizza, 2017; Fact Checker, 2017; 2018; Fandos; Landler, 2017; Bullock, 2018).

2. Arron Banks, de UKIP, en su campaña a favor del Brexit ya se dio cuenta de que la apelación a la emoción es la clave para tener éxito:

"Los hechos no funcionan. [...] Tienes que conectarte emocionalmente con la gente. Es el éxito de Trump" (Viner, 2016).

3. "Recuerdo haberle dicho alguna vez a Arthur Koestler que 'la historia se detuvo en 1936', ante lo cual él asintió, comprendiéndolo de inmediato. Ambos estábamos pensando en el totalitarismo en general, pero más particularmente en la Guerra Civil española. Ya de joven me había fijado en que ningún periódico cuenta nunca con fidelidad cómo suceden las cosas, pero en España vi por primera vez noticias de prensa que no tenían ninguna relación con los hechos, ni siquiera la relación que se presupone en una mentira corriente. Vi informar sobre grandiosas batallas cuando apenas se había producido una refriega, y silencio absoluto cuando habían caído cientos de hombres. Vi que se calificaba de cobardes y traidores a soldados que habían combatido con valentía, mientras que a otros que no habían visto disparar un fusil en su vida se los tenía por héroes de victorias inexistentes; y en Londres, vi periódicos que repetían estas mentiras e intelectuales entusiastas que articulaban superestructuras sentimentales sobre acontecimientos que jamás habían tenido lugar. [...] Estas cosas me parecen aterradoras, porque me hacen creer que incluso la idea de verdad objetiva está desapareciendo del mundo. A fin de cuentas, es muy probable que estas mentiras, o en cualquier caso otras equivalentes, pasen a la historia. [...] Sé que está de moda decir que casi toda la historia escrita es una sarta de mentiras. Estoy dispuesto a creer que la mayor parte de la historia es tendenciosa y poco sólida, pero lo que es característico de nuestro tiempo es la renuncia a la idea de que la historia se podría escribir con veracidad. En el pasado se mentía a sabiendas, o se maquillaba de forma inconsciente lo que se escribía, o se buscaba denodadamente la verdad, sabiendo muy bien que los errores eran inevitables; pero en cualquier caso se creía que 'los hechos' habían existido y que eran más o menos susceptibles de descubrirse" (Orwell, 2011 [1942]).

4. Según datos del Pew Research Center de finales de 2016, Facebook figuraba en tercer lugar entre los medios más consultados por los estadounidenses para información política (8\%), tras Fox News (19\%) y la CNN (13\%) (Gottfried; Barthel; Mitchell, 2017). A mediados de 2016, el 62\% de los estadounidenses adultos consumía noticias en redes sociales, siendo un $44 \%$ los que recurrían a Facebook (Gottfried; Shearer, 2016). Datos de agosto de 2017, ya superada la efervescencia electoral, mantienen la tendencia creciente: eran ya dos tercios (67\%) quienes recurrían a las redes sociales, con un repunte muy acusado del interés por la búsqueda de lo noticioso en Twitter, YouTube y Snapchat. Facebook seguía manteniendo una holgada ventaja sobre las otras, y además era la red que más se declaraba seguir para noticias de forma exclusiva (Shearer; Gottfried, 2017). Algunos autores, sin embargo, moderan el alcance electoral efectivo de las fake news propaladas a través redes sociales, y enfatizan el papel corrector de los medios tradicionales (Allcott; Gentzkow, 2017).

5. Junk news. Las noticias basura son "diversas formas de propaganda e información política ideológicamente extrema, hiper-partidista o conspirativa". Para estos investigadores, "la mayor parte de este contenido se produce de manera deliberadamente falsa. Busca persuadir a los lectores sobre las virtudes morales o los fallos de las organizaciones, causas o personas y presenta los comentarios como noticias. Este contenido lo producen organizaciones que no emplean periodistas profesionales, y el contenido utiliza técnicas de captación de atención, muchas fotografías, imágenes en movimiento, mayúsculas y minúsculas, ataques ad hominem, palabras e imágenes con carga emocional, generalizaciones inseguras y otras falacias lógicas" (Howard et al., 2017, p. 2).

\section{Referencias}

Allcott, Hunt; Gentzkow, Matthew (2017). "Social media and fake news in the 2016 election". Journal of economic perspectives, v. 31, n. 2, pp. 211-236.

https://www.jstor.org/stable/i40177264

Andrejevic, Mark (2013). Infoglut: How too much information is changing the way we think and know. New York: Routledge. ISBN: 9780415659086

Arendt, Hannah (2017). Verdad y mentira en la política. Barcelona: Página indómita [1972]. ISBN 9788494481673

Boorstin, Daniel J. (1992). The image: A guide to pseudo-events in America. New York: Vintage [1961]. ISBN: 9780 679741800 
Bounegru, Liliana; Gray, Jonathan; Venturini, Tommaso; Mauri, Michele (comps.) (2017). A field guide to fake news. Amsterdam: Public Data Lab; First Draft.

http://fakenews.publicdatalab.org

Bullock, James (2018). "The many times Donald Trump has attacked the media”. The guardian, 16 August.

https://www.theguardian.com/us-news/video/2018/aug/16/the-many-times-donald-trump-has-attacked-the-media-video

Bump, Philip (2017). “Donald Trump's inaugural crowds don't quite measure up to Barack Obama's". The Washington Post, 20 January.

https://goo.gl/YZyAYm

Cadwalladr, Carole; Graham-Harrison, Emma (2018). “Revealed: 50 million Facebook profiles harvested for Cambridge Analytica in major data breach". The guardian, 17 March.

https://www.theguardian.com/news/2018/mar/17/cambridge-analytica-facebook-influence-us-election

Cambridge Dictionary (2017). "Fake news". Cambridge Dictionary.

http://dictionary.cambridge.org/dictionary/english/fake-news

Chis, Teodora D. (2016). "The filter bubble: A constructivist approach". Perspectives in politics / Perspective politice, v. 9, n. 2, pp. 5-11.

http://perspective.politice.ro/article/filter-bubble-\%E2\%80\%93-constructivist-approach

Cillizza, Chris (2017). "Donald Trump's streak of falsehoods now stands at 33 days". The Washington Post, 21 Febr.

https://goo.gl/qjL1Ee

Cooke, Nicole A. (2017). "Posttruth, truthiness, and alternative facts: Information behavior and critical information consumption for a new age". Library quarterly: Information, community, policy, v. 87, n. 3, pp. 211-221.

https://www.journals.uchicago.edu/doi/pdfplus/10.1086/692298

Coromina, Òscar; Padilla, Adrián (2018). “Análisis de las desinformaciones del referéndum del 1 de octubre detectadas por Maldito Bulo". Quaderns del CAC, n. 44, pp. 17-26.

https://www.cac.cat/sites/default/files/2018-08/Q44_ES.pdf

D’Ancona, Matthew (2017). Post-truth: The new war on truth and how to fight back. Londres: Ebury Press. ISBN: 9781 785036873

Davies, William (2016). "The age of post-truth politics". The New York Times, 24 August. https://mobile.nytimes.com/2016/08/24/opinion/campaign-stops/the-age-of-post-truth-politics.htm/

Deleuze, Gilles (1990). Post-scriptum sur les sociétés de contrôle.

https://infokiosques.net/spip.php?article214

Del-Vicario, Michela; Vivaldo, Gianna; Bessi, Alessandro; Zollo, Fabiana; Scala, Antonio; Caldarelli, Guido; Quattrociocchi, Walter (2016). "Echo chambers: Emotional contagion and group polarization on Facebook". Scientific reports, n. 6, 37825. https://doi.org/10.1038/srep37825

Demirjian, Karoun (2018). "Russia favored Trump in 2016, Senate panel says, breaking with House GOP”. The Washington Post, 16 May.

https://goo.gl/quZN2L

Eisenberg, Michael B. (2008). “Information literacy: Essential skills for the information age”. Desidoc Journal of library \& information technology, v. 28, n. 2, pp. 39-47.

https://pdfs.semanticscholar.org/9598/2f446a1a4be155d66114d56ab34af5cf78ae.pdf

Elmborg, James (2006). “Critical information literacy: Implications for instructional practice". Journal of academic librarianship, v. 32, n. 2, pp. 192-199.

https://doi.org/10.1016/j.acalib.2005.12.004

Enguix-Oliver, Salvador (2017). “Impacto político e informativo de las redes sociales: esferas de actuación y comparación con los medios”. Anàlisi, n. 56, pp. 71-85.

https://doi.org/10.5565/rev/analisi.3090

European Commission (2018). A multi-dimensional approach to disinformation. Report of the independent High Level Group on Fake News and Online Disinformation. Luxembourg: Publications Office of the European Union.

https://doi.org/10.2759/0156

Fact Checker (2017). "100 days of Trump claims". The Washington Post, 29 April.

https://goo.gl/A5iKWF

Fact Checker (2019). "In 869 days, President Trump has made 10,796 false or misleading claims”. The Washington Post, 7 June. https://goo.gl/rGyjQa 
Fandos, Nicholas; Landler, Mark (2017). "Sean Spicer raises outcry with talk of Hitler, Assad and poison gas". The New York Times, 11 April.

https://goo.gl/RjY6XY

Faus, Joan (2018). "Facebook desbarata una campaña de desinformación a cuatro meses de las legislativas de EEUU”. EI país, 1 agosto. https://elpais.com/internacional/2018/07/31/actualidad/1533063515_375329.html

Fletcher, Richard; Cornia, Alessio; Graves, Lucas; Nielsen, Rasmus K. (2018). Measuring the reach of 'fake news' and online disinformation in Europe. Oxford: Reuters Institute for the Study of Journalism. https://goo.gl/nNTByn

Floridi, Luciano (2016). "Fake news and a 400-year-old problem: We need to resolve the 'post-truth' crisis". The guardian, 29 Nov. https://www.theguardian.com/technology/2016/nov/29/fake-news-echo-chamber-ethics-infosphere-internet-digital

Foucault, Michel (1977). "The political function of the intellectual”. Radical philosophy, v. 17, n. 13, pp. 126-133. https://www.radicalphilosophyarchive.com/article/the-political-function-of-the-intellectual

Frankfurt, Harry G. (2006). On bullshit. Sobre la manipulación de la verdad. Barcelona: Paidós [2005]. ISBN: 9788449318832 Fuchs, Christian (2014). Social media: A critical introduction. London: Sage. ISBN: 9781446257319

Gottfried, Jeffrey; Barthel, Michael; Mitchell, Amy (2017). "Trump, Clinton voters divided in their main source for election news". Pew Research Center, 18 January.

https://goo.gl/Ms82Lj

Gottfried, Jeffrey; Shearer, Elisa (2016). "News use across social media platforms 2016". Pew Research Center, 26 May. https://goo.gl/GSXFgT

Guess, Andrew; Nyhan, Brendan; Reifler, Jason (2018). Selective exposure to misinformation: Evidence from the consumption of fake news during the 2016 U.S. presidential campaign. European Research Council.

https://www.dartmouth.edu/ nyhan/fake-news-2016.pdf

Harsin, Jayson (2015). "Regimes of posttruth, postpolitics, and attention economy". Communication, culture \& critique, v. 8, n. 2, pp. 327-333. https://doi.org/10.1111/cccr.12097

Harsin, Jayson (2018). "A critical guide to fake news: From comedy to tragedy". Pouvoirs. Revue française d'études constitutionnelles et politiques, n. 164, pp. 99-119.

http://www.revue-pouvoirs.fr/A-Critical-Guide-to-Fake-News-From.html

Howard, Philip N.; Bolsover, Gillian; Kollanyi, Bence; Bradshaw, Samantha; Neudert, Lisa-Maria (2017). "Junk news and bots during the U.S. election: What were Michigan voters sharing over Twitter?". The computational propaganda project. https://comprop.oii.ox.ac.uk/research/working-papers/junk-news-and-bots-during-the-u-s-election-what-were-michiganvoters-sharing-over-twitter

Howard, Philip N.; Kollanyi, Bence (2016). “Bots, \#Strongerin, and \#Brexit: Computational propaganda during the UK-EU referendum".

https://doi.org/10.2139/ssrn.2798311

Howard, Philip N.; Woolley, Samuel; Calo, Ryan (2018). "Algorithms, bots, and political communication in the US 2016 election: The challenge of automated political communication for election law and administration". Journal of information technology and politics, v. 15, n. 2, pp. 81-93.

https://doi.org/10.1080/19331681.2018.1448735

Ireton, Christine; Posetti, Julie (eds.) (2018). Journalism, 'fake news' \& disinformation: A handbook for journalism education and training. Paris: Unesco.

https://en.unesco.org/node/295873

Jiménez-Cano, Rosa (2017a). "Facebook estrena un sistema para detectar las noticias falsas”. El país, 6 abril. https://goo.gl/HKZ72Y

Jiménez-Cano, Rosa (2017b). “Facebook cooperará en la investigación por la trama rusa”. El país, 22 septiembre. https://goo.gl/CR4p41

Jiménez-Cano, Rosa (2017c). “La propaganda de la trama rusa llegó a 10 millones de perfiles en Facebook”. El país, 3 octubre. https://goo.gl/G6ozZR

Kennedy, Jenny (2013). "Rhetorics of sharing: Data, imagination, and desire". In: Lovink, Geert; Rasch, Miriam (eds.). Unlike us reader: Social media monopolies and their alternatives. Amsterdam: Institute of Network Cultures, pp. 127136. ISBN: 9789081857529 
Keyes, Ralph (2004). The post-truth era: Dishonesty and deception in contemporary life. New York: St. Martin's Press. ISBN: 9781429976220

Kosiński, Michal; Stillwell, David; Graepel, Thore (2013). "Private traits and attributes are predictable from digital records of human behavior". In: Proceedings of the National Academy of Sciences of the USA, v. 110, n. 15, pp. 5802-5805. https://doi.org/10.1073/pnas.1218772110

Koyré, Alexandre (2015). La función política de la mentira moderna. Madrid: Pasos Perdidos. ISBN: 9788494343445

Lennon, J. Michael (ed.) (2014). Selected letters of Norman Mailer. New York: Random House. ISBN: 9780812986105

Mailer, Norman (1973). Marilyn: A biography. New York: Grosset \& Dunlap. ISBN: 9780448010298

Marda, Vidushi; Milan, Stefania (2018). Wisdom of the crowd: Multistakeholder perspectives in the fake news debate. Report by the Internet Policy Observatory at the Annenberg School, University of Pennsylvania.

https://papers.ssrn.com/sol3/papers.cfm?abstract_id=3184458

Mannheim, Karl; Wirth, Louis; Shils, Edward (1936). Ideology and utopia. Londres: K. Paul, Trench, Trubner \& Co. ISBN: 9780710031235

Mars, Amanda; Jiménez-Cano, Rosa (2017). “Las grandes tecnológicas admiten que toneladas de propaganda rusa invadieron sus plataformas durante las elecciones". El país, 31 octubre.

https://goo.gl/1kxxC4

McNair, Brian (2018). Fake news, falsehood, fabrication, and fantasy in journalism. London: Routledge. ISBN: 9781 138306790

Miller, David (ed.) (2004). Tell me lies: Propaganda and media distortion in the attack on Iraq. London: Pluto Press. ISBN: 9780745322018

Moses, Lucia (2016). "43 percent of social media users don't know where the stories they read originally appeared". Digiday, 13 May.

https://goo.gl/QF6MTX

Nelson, Jakob L.; Taneja, Harsh (2018). "The small, disloyal fake news audience: The role of audience availability in fake news consumption". New media \& society, v. 20, n. 10, pp. 3720-3737.

https://doi.org/10.1177/1461444818758715

Nyhan, Brendan; Reifler, Jason (2010). "When corrections fail: The persistence of political misperceptions". Political behaviour, v. 32, n. 2, pp. 303-330.

https://link.springer.com/article/10.1007/s11109-010-9112-2

Oremus, Will (2016). "How many people really get their news from Facebook?". Slate, 20 Dec.

http://www.slate.com/articles/technology/technology/2016/12/how_many_people_really_get_their_news_from_ facebook.html

Orwell, George (1980). "Looking back on the Spanish War" [1942]. En: Orwell, Sonia; Angus, Ian (eds.). The collected essays, journalism and letters. Volume 2: My country right or left. London: Penguin, pp. 290-298. ISBN: 9780140187120

Oxford English Dictionary (2016). "Word of the Year 2016 is...". Oxford English Dictionary.

https://en.oxforddictionaries.com/word-of-the-year/word-of-the-year-2016

Pariser, Eli (2011). The filter bubble. New York: Penguin. ISBN: 9780141969923

Pennycook, Gordon; Rand, David G. (2018). "Lazy, not biased: Susceptibility to partisan fake news is better explained by lack of reasoning than by motivated reasoning". Cognition, v. 188, pp. 39-50.

https://doi.org/10.1016/j.cognition.2018.06.011

Petit, Martí (2018). "Por una crítica de la razón algorítmica: Estado de la cuestión sobre la inteligencia artificial, su influencia en la política y su regulación". Quaderns del CAC, n. 44, pp. 5-15.

https://www.cac.cat/sites/default/files/2018-08/Q44_ES.pdf

Pickard, Victor (2016). "Media and politics in the age of Trump". Origins: Current events in historical perspective, v. 10, n. 2. https://origins.osu.edu/article/media-and-politics-age-trump

Porter, Ethan (2017). "Facts matter, and people care: An empirical perspective". En: Powers, Shawn; Kounalakis, Markos (eds.). Can public democracy survive the internet? Bots, echo chambers, and disinformation. Washington: U.S. Advisory Commission on Public Diplomacy (Department of State), pp. 51-59.

https://www.hsdl.org/?abstract\&did=800873 
Powers, Shawn; Kounalakis, Markos (eds.) (2017) Can public democracy survive the internet? Bots, echo chambers, and disinformation. Washington: U.S. Advisory Commission on Public Diplomacy (Department of State). https://www.hsdl.org/?abstract\&did=800873

RAE (2017). "Posverdad". Diccionario de la lengua española.

http://dle.rae.es/?id=TqpLeOm

Rodríguez-Ferrándiz, Raúl (2018a). Máscaras de la mentira: el nuevo desorden de la posverdad. Valencia: Pre-Textos. ISBN: 9788417143299

Rodríguez-Ferrándiz, Raúl (2018b). “Fakebook: miénteme al oído”. La maleta de Portbou, n. 31, pp. 11-15. https://lamaletadeportbou.com/fakebook-mienteme-al-oido

Rosenberg, Matthew; Confessore, Nicholas; Cadwalladr, Carole (2018). "How Trump consultants exploited the Facebook data of millions". The New York Times, 17 March.

https://www.nytimes.com/2018/03/17/us/politics/cambridge-analytica-trump-campaign.html

Shearer, Elisa; Gottfried, Jeffrey (2017). "News use across social media platforms 2017". Pew Research Center, 7 Sept. https://goo.gl/LNhaQF

Silverman, Craig (2016). "This analysis shows how viral fake election news stories outperformed real news on Facebook". BuzzFeed news, 16 Nov.

https://goo.gl/WNY7UV

Silverman, Craig; Singer-Vine, Jeremy (2016). "Most Americans who see fake news believe it, new survey says". BuzzFeed news, 6 Dec. https://goo.gl/B46kCK

Stanley, Jason (2017). "In defense of truth, and the threat of disinformation". In: Powers, Shawn; Kounalakis, Markos (eds.). Can public democracy survive the internet? Bots, echo chambers, and disinformation. Washington: U.S. Advisory Commission on Public Diplomacy (Department of State), pp. 71-75.

https://www.hsdl.org/?abstract\&did=800873

Sulleyman, Aatif (2017). "Here is Facebook's guide to fake news". The independent, 9 May. http://www.independent.co.uk/news/facebook-fake-news-guide-articles-curate-stop-take-down-lies-russia-donald-trumpus-politics-a7726111.html

Tesich, Steve (1992). "A government of lies". The nation, 13 June. https://goo.gl/dzEZqi

Thompson, Mark (2016). Enough said: What's gone wrong with the language of politics?. New York: St. Martin's Press. ISBN: 9781250059574

Ufarte-Ruiz, María-José; Peralta-García, Lidia; Murcia-Verdú, Francisco-José (2018). “Fact checking: un nuevo desafío del periodismo". El profesional de la información, v. 27, n. 4, pp. 733-741.

https://doi.org/10.3145/epi.2018.jul.02

Van-Dijck, José (2013). The culture of connectivity, Oxford: Oxford U.P. ISBN: 9780199970773

https://doi.org/10.1093/acprof:oso/9780199970773.001.0001

Viner, Katharine (2016). "How technology disrupted the truth". The guardian, 12 July. https://www.theguardian.com/media/2016/jul/12/how-technology-disrupted-the-truth

Wardle, Claire; Derakhshan, Hossein (2017). Information disorder: Toward an interdisciplinary framework for research and policy making. Council of Europe Report DGI(2017)09. Bruselas: Consejo de Europa.

https://edoc.coe.int/en/media/7495-information-disorder-toward-an-interdisciplinary-framework-for-research-andpolicy-making.html

White, Aidan (ed.) (2017). Ethics in the news. Report on challenges for journalism in the post-truth era. Londres: Ethical Journalism Network.

https://ethicaljournalismnetwork.org/wp-content/uploads/2017/01/ejn-ethics-in-the-news.pdf

Wingfield, Nick; Isaac, Mike; Benner, Katie (2016). "Google and Facebook take aim at fake news sites". The New York Times, 14 Nov.

https://nyti.ms/2x5GmK7

Woolley, Samuel (2017). "Computational propaganda and political bots: An overview". In: Powers, Shawn; Kounalakis, Markos (eds.). Can public democracy survive the internet? Bots, echo chambers, and disinformation. Washington: U.S. Advisory Commission on Public Diplomacy (Department of State), pp. 13-17.

https://www.hsdl.org/?abstract\&did $=800873$ 